\title{
BISTATIC AND MULTISTATIC RADAR SENSORS FOR HOMELAND SECURITY
}

\author{
C.J. Baker - H.D. Griffiths \\ University College London \\ U.K.
}

\begin{abstract}
The separation of transmitter and receiver in bistatic and multistatic radar sensors offers the system designer new and additional degrees of freedom to tailor solutions to specific applications. The receivers may be passive and hence largely immune to jamming. Passive systems that also use 'illuminators of opportunity' do not have to provide a potentially expensive transmitter. Multiple transmitters and or receivers can improve sensitivity, coverage, and importantly improve the opportunity to acquire a line of site to the target (without which detection is impossible). These advantages make this form of radar attractive for a variety of applications, many of which fit well with the needs of homeland security. Equally, however, the additional complexity of having a number of separated transmitters and receivers brings about new challenges that require careful understanding if these forms of sensors are to be routinely adopted for operational use.

In this chapter the role of active and passive techniques as a support to homeland security is explored. The essentials of bistatic and netted radar are introduced which enables the relative strengths and weaknesses of these approaches to be outlined. In this way a foundation is provided against which a variety of potential applications may be explored.
\end{abstract}

Keywords:

radar; bistatic radar; multistatic radar; ambiguity function; parasitic radar; forward scatter; Babinet's principle; passive coherent location; netted radar.

\section{Introduction}

Radar has long been used in a variety of military and civilian applications and has become an essential component of current defensive systems. The chief reason for this is an ability to survey wide areas rapidly during the day or at night and in all weather conditions. It is 
the only sensor able to do this. Many countries have a network of civil aviation radars that often form a part of a wider air defence capability that is able to detect aircraft out to ranges of hundreds of $\mathrm{km}$. These networks are specifically designed to ensure early warning against potentially hostile threat targets. In a similar manner coastal shorelines are monitored also using a combination of civilian coastguard and military maritime systems.

However, the range and nature of potential threat targets is becoming ever more diverse. For example targets are becoming faster, more agile, stealthier and can occur in many guises. The tragic events of $9 / 11$ are of course but one example of how the meaning of legitimate 'targets' has changed irrevocably. Others might include missile attack, uavs (including micro-lights), small high speed boats (including jetskis). In fact the potential range of threat targets is almost unlimited. This means that the source of attack can emanate in a much wider variety of new and different forms. These are not necessarily well dealt with by current conventional radar systems and alternatives merit evaluation.

Here we concern ourselves with bi and multistatic radar concepts, largely, although not exclusively, as an appliqué fit to existing systems. One example might be the augmentation of current air defence systems to ensure that coverage is extended into areas not well catered for by current systems (such as low level flight paths). In this way high value or physically vulnerable assets (such as nuclear power plants) may be afforded improved protection. Bistatic and multistatic radar systems have a number of advantages that make them potentially very well suited to these types of applications. They don't necessarily require expensive transmitters and are relatively immune to physical and electronic attack due to their inherent passivity and their distributed nature. They are also better able to detect stealthy targets which have been designed only to present a small cross-section to monostatic radar. However, this tends to come at the cost of increased system complexity. Nevertheless tradeoffs between performance and complexity can show worthwhile benefits.

Here we introduce the principles of bistatic and multistatic radar. Firstly, we examine what is meant by these terms and then go on to develop the fundamental relationships that govern performance in terms of sensitivity, coverage, range resolution, Doppler resolution and target location accuracy. This provides the essential information necessary to understand the advantages and disadvantages of bistatic and multistatic radar operation for candidate applications. More detail can be found in the excellent text of Willis [1]. 


\section{Definitions}

A survey of the literature reveals that definitions of bistatic and multistatic radar are quite widely varying with no universal acceptance of single descriptions. The IEEE defines bistatic radar as 'a radar system that uses antennas at different locations for transmission and reception'. The distance of separation between the two is referred to as the 'baseline' range. However, there is no stipulation as to how far apart the two antennas should be. Clearly if they are near co-located, i.e. if the baseline is very small, then the system approximates monostatic radar. Very small baselines are sometimes used in cw systems where there is a need to minimise the likelihood of the transmitted signal being received directly, thus masking the presence of real targets. Suppression of the directly received signal is difficult to achieve via a single antenna typically used in monostatic radar. Here we do not consider such a system to be bistatic. If a further antenna (either transmitting or receiving or both) is added to the bistatic pair then this might be termed multistatic radar. However, other terminology often includes 'netted radar', 'multi site radar' and 'distributed radar'. The distinctions between these are, at

best, somewhat blurred. Here we will use the term multistatic to mean any system comprising a bistatic pair augmented by an additional antenna (tranmit or receive). These two labels are not entirely satisfactory as there is no reason why the antennas need be 'static'!!

A particular variant on the bistatic and multistatic themes is Passive Coherent Location (PCL). This is normally taken to mean a system where transmissions are provided by a third party and only the receiver is formerly part of the design. This is sometimes also referred to as a 'hitch hiking' mode of operation. It is also usually referred to as an example of bistatic radar although, as we shall see later, many transmissions can be used from a variety of transmitter sites thus making an example of multistatic radar.

\section{Bistatic essentials}

In this section we review the fundamental building blocks of bistatic radar emphasising similarities and differences with the more usual monostatic counterpart. Figure 1 shows a typical bistatic geometry with clear separation of the transmitter and receiver.

It has two geometrical characteristics which differentiate it from conventional monostatic systems. These are:

- the transmitter receiver separation and ;

- the transmitter-target-receiver triangulation. 


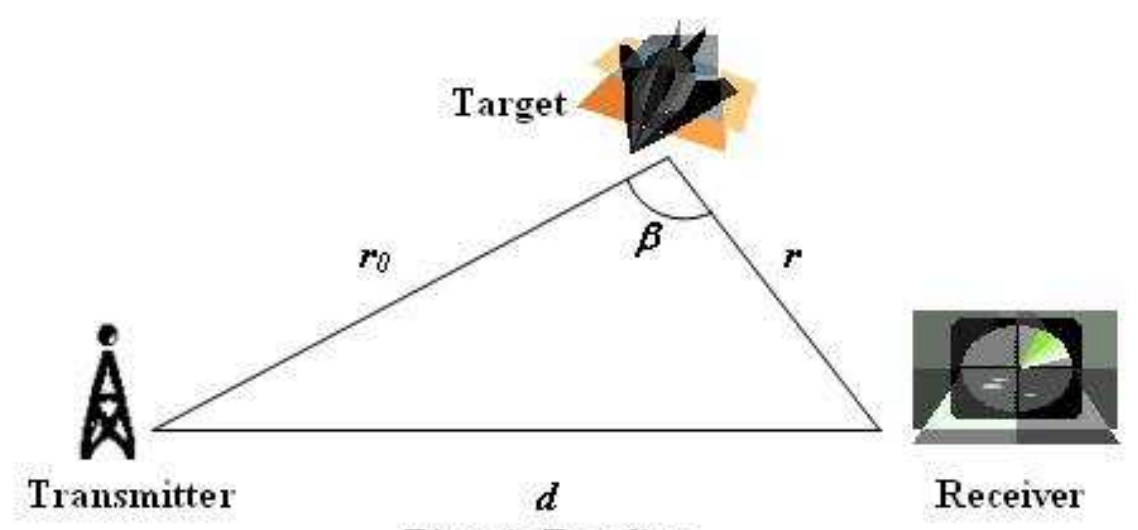

Bistatic Baseline

Figure 1. Bistatic geometry

The distance $d$ represents the bistatic baseline. $r_{O}$ is the transmitter to target separation and $r$ is the target to receiver separation. $\beta$ is referred to as the bistatic angle.

The baseline distance is usually fixed or slowly varying. The system performance depends to a large extent on the target position relative to the bistatic baseline and hence triangulation. Three target position areas can be described with substantially different characteristics. There is the broadside area with a bistatic angle less that $180^{\circ}$ which provides the most common form of bistatic radar. There is also the baseline area with a bistatic angle equal to $180^{\circ}$. This situation corresponds to the 'forward scatter' geometry and has several implications which will be discussed later. The final area is the extended baseline behind both the transmitter and receiver. This obeys quasi-bistatic characteristics.

The bistatic radar range equation is given by

$$
\frac{P_{r}}{P_{n}}=\frac{P_{t} G_{t} G_{r} \lambda^{2} L_{p t} L_{p r} \sigma_{b}}{(4 \pi)^{3} r_{0}^{2} r^{2} k T_{0} B F}
$$

where

$P_{t}$ is the transmitter power in Watts

$G_{t}$ is the gain of the transmitter antenna

$G_{r}$ is the gain of the receiving antenna

$\lambda$ is the radar wavelength in metres

$L_{p t}$ is the loss from transmitter to target $(\leq 1)$

$L_{p r}$ is the loss from target to receiver $(\leq 1)$ 
$r_{o}$ is the distance between the transmitter and target in metres

$r$ is the distance between the receiver and target in metres

$k$ is the Boltzmann's constant

$T_{0}$ is the noise reference temperature in kelvin

$B$ is the receiver bandwidth in $\mathrm{Hz}$

$F$ is the receiver noise figure

$\sigma_{b}$ is the bistatic Radar Cross Section in $m^{2}$.

The main difference between bistatic and monostatic radar is the separation of transmitter and receiver ranges. These determine the bistatic geometry. From the bistatic geometry, it can be observed that targets of constant bistatic range are described by ellipses with the transmitter and receiver as the two foci. We note that in monostatic radar these are, of course, circles. In bistatic radar these ellipses are the same as the contours of zero Doppler. The contours of maximum Doppler shift form hyperbolae as they must also cross these ellipses orthogonally. In monostatic radar the orthogonal condition again holds but this time leads to a series of lines emanating radially out from the co-located transmitter receiver pair. In bistatic radar a moving target will not present zero Doppler to two receiving sites simultaneously. This can usefully be exploited in multistatic radar systems.

Contours of constant signal to noise ratio follow the lines of ovals of Cassini as the signal to noise ratio is inversely proportional to the product of the squares of the transmitter to target and target to receiver ranges. In monostatic radar contours of constant signal to noise ratio are circles.

The bistatic radar cross section of the target is not necessarily the same as the monostatic one. For small bistatic angles of less than approximately $5^{\circ}$, the bistatic RCS of a complex target is equal to the monostatic RCS measured on the bisector of the bistatic angle at a frequency lower by a factor of $\cos (\beta / 2)$. Also, when operating in the broadside area, bistatic radar may be well suited to detecting stealthy targets. This is because a target is very unlikely to present a low bistatic cross section to two receiving sites simultaneously. This potentially makes the detection of stealthy targets easier as the reflections from it in other directions will be detected by bistatic receivers.

When a target crosses the baseline of a bistatic radar the RCS can be greatly enhanced. This is due to the forward scatter phenomenon or "Babinets" principle. Here the RCS of a target at the bistatic baseline is calculated from

$$
\sigma_{b}=\frac{4 \pi A^{2}}{\lambda^{2}}
$$


where

$A$ is the geometric area of the target in $m^{2}$

$\lambda$ is the radar wavelength in metres.

For a sphere of radius a metres, the monostatic RCS is equal to the projected area of a sphere given by $\pi a^{2}$. Considering a sphere with monostatic RCS equal to $0.25 \mathrm{~m}^{2}$ and at a wavelength equal to $0.1 \mathrm{~m}$, the forward scatter RCS is:

$$
\sigma_{b}=\frac{4 \pi(0.25)^{2}}{(0.1)^{2}}=78.5 m^{2}
$$

This corresponds to an enhancement of $25 \mathrm{~dB}$. The forward scatter RCS will decrease as the bistatic angle decreases and ultimately reaches the monostatic RCS in the limit where the angle is equal to zero. Nevertheless, significant RCS enhancement is generally achieved at bistatic angles of $165^{\circ}$. An important factor is that the forward scatter RCS does not depend on material composition. As a result, bistatic radars operating in the forward scatter region may be able to detect stealthy targets and will give appreciable forward scatter RCS despite their designed low monostatic RCS. In addition it should be noted that the angular width of scattering is a function of the wavelength and hence favours low frequencies.

Bistatic clutter is a poorly understood branch of radar and few measurements have been undertaken to help develop useful models. This is a subject that requires further and quite urgent research.

There are also some important differences in the technology required to realise bistatic radar. In monostatic radar synchronisation between transmission and reception is done via a stable source, usually a local oscillator. In bistatic radar the separation of transmitter and receiver makes this much more difficult. An equivalent situation has to be achieved and this is done either via synchronised atomic clocks, a signal such as GPS or by reception of a reference signal received directly from the transmitter. The latter technique is typically used in PCL systems and we shall return to this later.

Another important difference between bistatic and monostatic radar is that a directional receive antenna must scan at a non uniform rate to follow the position of the transmitted signal through space, a process known as pulse chasing. This can be very challenging for designs based upon mechanical scanning and hence an alternative is to use one or more electronically agile beams as in phased array radar. Such phased array antennas can be expensive and in some applications will prohibit the use of the bistatic technique. 


\section{Passive Coherent Location (PCL)}

This section looks at the intriging concept of utilising illuminators of opportunity to give a completely passive bistatic radar system. These are signals such as existing radars, communications, navigation and broadcast transmissions which happen to be in existence. Their characteristics are governed by their own missions and hence their waveforms will not necessarily have the ideal parameters for a given bistatic radar system. Nevertheless, the advantage of using such signals is their availability, fixed position and somewhat known characteristics which make building and optimising a passive receiver less complex. PCL systems can use transmissions from multiple nearby sources, hence making them an example of multistatic radar. For now, however, we can assume that they have (for any given single transmitter) the same bistatic geometry introduced in the previous section.

There has recently been an upsurge of interest in PCL radar systems that exploit illuminators of opportunity. An additional attraction is that this can dramatically reduce the costs of the system hardware. The rapid growth in number of RF emissions for TV and radio broadcasts as well as terrestrial and space based communications has resulted in a wide range of signal types available for exploitation by passive radar. Further, many such transmissions are at VHF and UHF frequencies, which allows these parts of the spectrum not normally available for radar use, and at which stealth treatment of targets may be less effective, to be used. However, the location of the transmitter and the form of the transmission to be exploited is no longer under the control of the radar designer. The multiplicity of transmissions from both terrestrial [2] and space based sources $[3,4]$ provide spatial and frequency diversity and can be exploited to further improve detection performance. Examples of reported operational systems include the Lockheed Martin 'Silent Sentry' system for air and space surveillance [2], the Roke Manor Research CELLDAR system for air target detection [5] and the Manastash Ridge radar for atmospheric and ionospheric studies [6]. Other reported experimental systems include those proposed by Dynetics [7] and UCL [8-10]. Applications include air-space surveillance $[2,5]$, maritime surveillance [10], atmospheric studies [6], ionospheric studies [6], oceanography [11], mapping lightning channels in thunderstorms [12] and monitoring radioactive pollution [13]. There have also been recent reports of algorithm development for interferometry [16], target tracking [17] and target classification $[17,18]$. This range and diversity of systems and applications is indicative of the increasing importance of this form of sensor system. 
The transmit power $P_{t}$ is substantial for many passive radar sources, since broadcast and communications receivers often have inefficient antennas and poor noise figures and the transmission paths are often far from line-of-sight; thus the transmit powers have to be significantly higher to overcome the inefficiencies and losses. In the UK, the highest power FM radio transmissions are $250 \mathrm{~kW}$ (ERP) per channel, with many more of lower power [19]. The highest power analogue TV transmissions are $1 M W$ (ERP) per channel [19]. These are omnidirectional in azimuth, and are sited on tall masts on high locations to give good coverage. The vertical-plane radiation patterns are tailored to avoid wasting too much power above the horizontal.

GSM cellphone transmissions in the UK are in the $900 \mathrm{MHz}$ and $1.8 \mathrm{GHz}$ bands. The modulation format is such that the downlink and uplink bands are each of $25 \mathrm{MHz}$ bandwidth, split into 125 FDMA channels each of $200 \mathrm{kHz}$ bandwidth, and a given basestation will only use a small number of these channels. Each channel carries 8 signals via TDMA, using GMSK modulation. Third generation (3G) transmissions are in the $2 \mathrm{GHz}$ band, using CDMA modulation over $5 \mathrm{MHz}$ bandwidth. The radiation patterns of cellphone basestation antennas are typically arranged in $120^{\circ}$ azimuth sectors, and shaped in the vertical plane again to avoid wasting power. The patterns of frequency re-use means that there will be cells using the same frequencies within quite short ranges. Licensed ERPs are typically in the region of $400 \mathrm{~W}$, although in many cases the actual transmit powers are lower. The OFCOM sitefinder website [20] gives details of the location and operating parameters of each basestation throughout the UK, and an example of the information provided by this website is shown in Table 1 .

In all cases it is necessary to consider the power in the portion of the signal spectrum used for passive radar purposes, which may not be the same as the power of the total signal spectrum. For example the ambiguity properties of the full signal may not be as favourable as those of a portion of the signal. This is the case for an analogue television transmission; the full signal has pronounced ambiguities associated with the $64 \mu s$ line repetition rate, but better ambiguity performance may be realised by taking just a portion of the signal spectrum at the expense of reduced signal power.

In PCL systems care must be taken to ensure that the signal received directly from the transmitter does not compete with and swamp that from the target. Typically this will be the case unless measures are taken to suppress the direct signal occurring in the indirect channel. We can formulate a simple expression for the amount of direct signal suppression required by calculating the ratio of the indirect received sig- 
nal to the direct signal and requiring this to be at least the same value as that used to compute the maximum detection range. We make the simple assumption that a target can be seen above this level of direct signal breakthrough and hence that it approximates to the highest level of interference that is tolerable for single 'pulse-like' detection. There is, however, no benefit from integration as the direct leakage will also integrate up, and this may lead to a more stringent requirement needing to be set in practice. This places the direct leakage signal at the same level as the noise floor in the receiver and hence it has the attractive feature of proving equivalent performance to 'single-pulse' detection. Thus to achieve adequate suppression and hence maintenance of full system dynamic range the direct signal must be cancelled by an amount given by the magnitude of the ratio of the indirect and directly received signals, e.g.

$$
\frac{P_{r}}{P_{d}}=\frac{r_{b}^{2} \sigma_{b}}{4 \pi r_{1}^{2} r_{2}^{2}}
$$

where $P_{r}$ is the target echo signal, $P_{d}$ is the direct signal, and $r_{b}$ is the transmitter-to-receiver range (bistatic baseline). This expression is indicative only and strictly speaking the direct signal should be below that of the noise floor after integration, if integration is employed.

There are several techniques that may be used to suppress this leakage. These include: (i) physical shielding, (ii) Doppler (Fourier) processing, (iii) high gain antennas, (iv) sidelobe cancellation, (v) adaptive beamforming and (vi) adaptive filtering. Each one of these techniques will provide different suppression characteristics over the $(\theta, f)$ plane - thus physical shielding or beamforming techniques will provide suppression as a function of $\theta$; Doppler processing or adaptive filtering will provide suppression as a function of $f$. The combination of high gain antennas and adaptive beam-forming also enables multiple simultaneous transmissions to be exploited. The Manastash Ridge radar [6] provides an example of the use of physical shielding; in this case suppression is achieved by siting the receiver on the other side of a large mountain which provides the screening. In other cases some simpler more localised methods may be used such as appropriate deployment of absorbing material (RAM). For the detection of moving targets Doppler or Fourier processing will automatically improve dynamic range, as the direct signal leakage will only occur at DC (with some spill over). However it should be noted that significant sidelobe leakage due to inadequate suppression of very strong directly received signals will reduce the gain from Fourier processing and hence impair dynamic range. 
For the example considered here, the transmitter at Wrotham in the south-east of England is taken together with a receiver sited at the Engineering building of UCL. The transmitted power is $250 \mathrm{~kW}$ and broadcasts are made in the frequency range 89.1-93.5MHz. Figure 2 shows a plot of the detection range; the contour represents a signal-to-noise ratio of $15 \mathrm{~dB}$ (and this value is used for all subsequent figures of this type). The modulation bandwidth is taken as $55 \mathrm{kHz}$. A signal-to-noise ratio of $15 \mathrm{~dB}$ or greater is maintained out to a range of nearly $30 \mathrm{~km}$. This performance is constrained by the effective noise figure of the receiver, and better performance would be obtained with better suppression of direct signal and noise. It should be noted that the power emitted by transmitters across the UK varies from as little as $4 \mathrm{~W}$ to a maximum of $250 \mathrm{~kW}$ and of course this variation has to be carefully factored in to performance predictions.

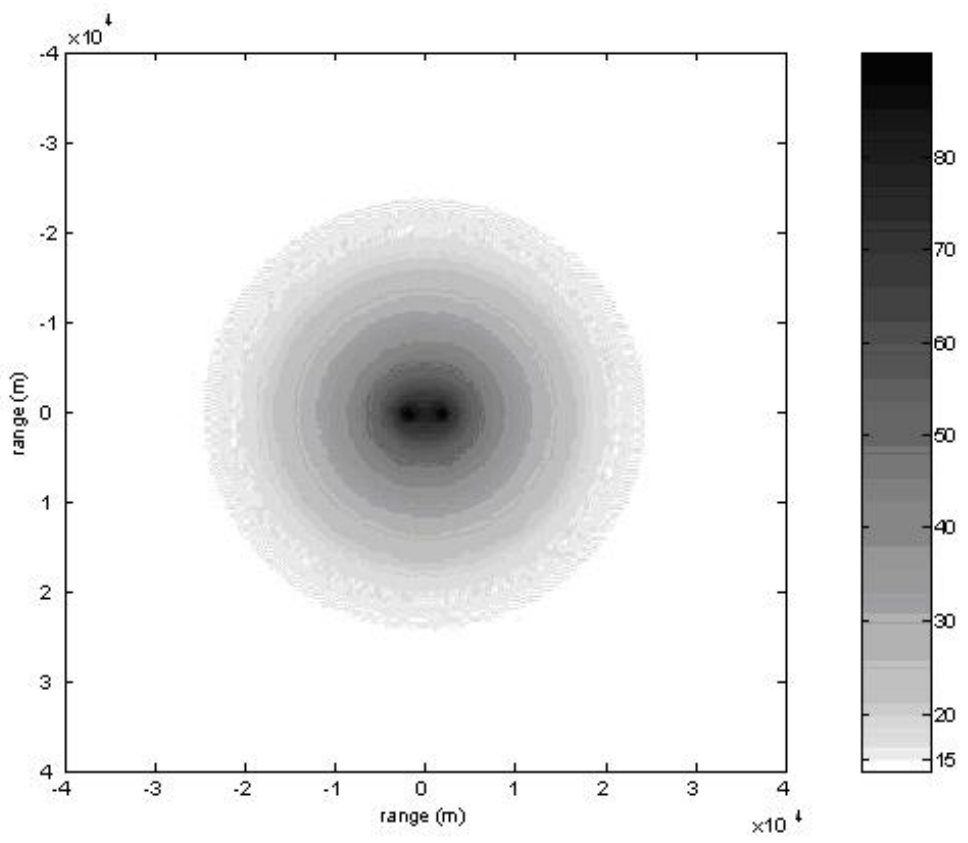

Figure 2. Detection range for an FM radio transmitter at Wrotham in south-east England and a receiver at UCL. The solid contour represents a signal-to-noise ratio of $15 \mathrm{~dB}$.

Range resolution is the ability of a radar system to distinguish two closely spaced targets at differing ranges. In monostatic radar this is 
primarily a function of pulse length or modulation bandwidth. Azimuth and elevation resolutions distinguish between two targets in angle space and are determined by antenna dimensions and operating wavelength. Together these specify the three-dimensional spatial resolution of any radar system. Doppler resolution is the ability to distinguish two targets by virtue of their differing velocities. In a monostatic radar system this is primarily a function of wavelength, waveform and target illumination time. It is important to understand the range and Doppler resolution of any radar system in order that overall performance may be reliably estimated. Examples might include ensuring that tracking performance is adequate for a particular application or that image quality metrics result in two-dimensional target signatures suitable for further processing tasks such as classification. In monostatic radar systems these parameters are routinely established as part of the design process and can be easily pre-determined by the radar designer to be tailored to the chosen application. However, in bistatic radar systems and more particularly in Passive Coherent Location (PCL) systems they cease to be of a routine nature and considerable care needs to be exercised in these aspects of design. Indeed in the case of PCL the lack of design control over the form, nature and origin of the transmitted waveform seems to imply severe restrictions. In practice, however, there are more freedoms than might be apparent at first sight as usually more than one transmitter may be used at any given instant of time. This is an aspect not exploited in monostatic radar systems. Nevertheless, separation of the transmitter and receiver and the time varying properties of qualifying illuminations of opportunity do result in important differences that must be thoroughly understood if PCL system design methods are to evolve to similar levels of maturity to that of monostatic radar. In this chapter we analyse these fundamental aspects of PCL radar design that determine subsequent performance. Practical measurements of transmissions of opportunity show detailed, time-dependent, behaviours that require careful consideration when developing an overall system design.

Specifically the ambiguity function has long been used to evaluate range and Doppler resolution as well as range and Doppler ambiguity. However, it was developed to capture these aspects of performance for monostatic radar systems only. Here we review the ambiguity function and in particular its bistatic formulation. This highlights the importance of system geometry with respect to target position. Results of the bistatic 'self-ambiguity' of 'on-air' signals are used to demonstrate waveform variability and its effect on range and Doppler resolution as well as detection performance. These give the best possible range and Doppler resolutions and are geometry independent. The self-ambiguity 
function is equivalent to the transmitter and receiver being co-located, i.e., the monostatic case. The more general description of ambiguity in a bistatic system introduces a geometrical dependence between the transmitter, receiver and target that determines the range and Doppler resolutions.

The ambiguity function represents the output of a matched filter and may be written as

$$
\left|\psi\left(R_{R}, f_{d}\right)^{2}\right|=\left|\int_{-\infty}^{+\infty} s_{t}(t) \cdot s_{t}^{*}\left(t+R_{R}\right) \cdot \exp \left[j 2 \pi f_{d} t\right] d t\right|^{2}
$$

where $\psi\left(R_{R}, f_{d}\right)$ is the ambiguity response at delay range and Doppler and $s_{t}(t)$ is the directly received transmitted signal

Computation of this function results in a three-dimensional plot for which one axis is time delay (or range), the second is Doppler frequency or radial velocity and the third is the output power of the matched filter (usually normalised to unity). The extent of the ambiguity function peak in the $T_{R}$ and the $f_{d}$ dimensions determines the range and Doppler resolutions respectively. As we are using the directly received signal only we term this "self-ambiguity" as there is no inclusion of any system geometry dependence on the transmitter and receiver locations.

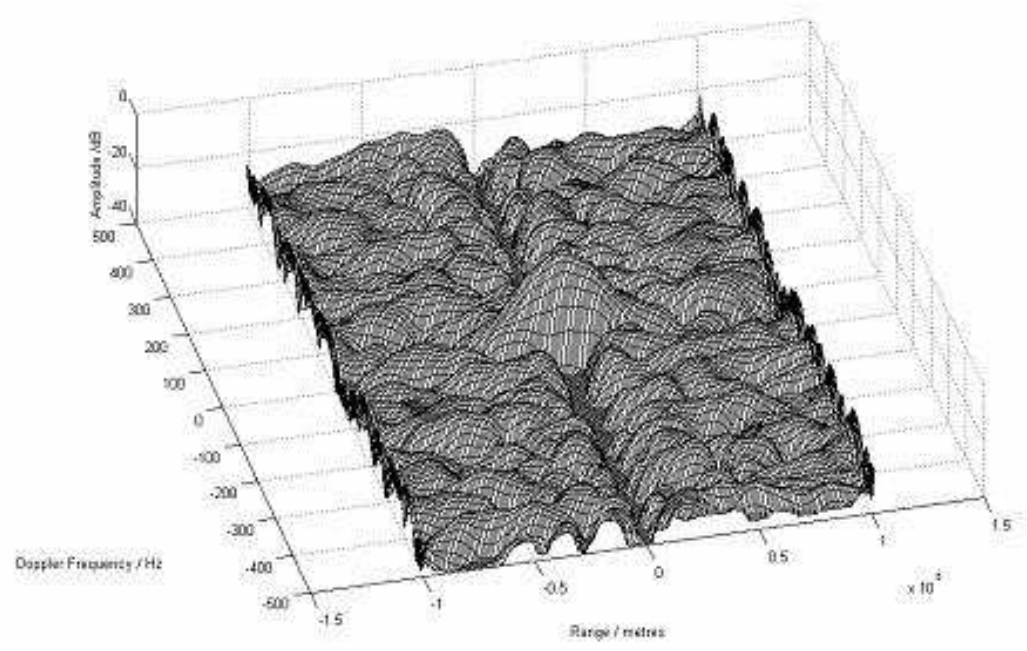

Figure 3. The ambiguity function for a BBC radio 4 transmission at $93.5 \mathrm{MHz}$.

An example self ambiguity function is shown in Figure 3. The sample length taken was $80 \mathrm{~ms}$ of stereo signal which was sampled after apply- 
ing a filter of bandwidth $300 \mathrm{kHz}$. Figure 3 shows an un-weighted selfambiguity function for a BBC Radio 4 transmission for which the signal comprises speech (in this instance an announcer reading the news). The peak of the ambiguity function is reasonably well defined but a lot of fine, semi-random structure can be seen in the regions away from the main peak which are a function of the detailed modulation present in this component of the waveform. This does not show pure noise-like behaviour but is consistent with the correlation that might be expected in a speech type signal. Cuts taken at zero range and Doppler are also shown in Figure 4 to demonstrate the range and Doppler resolutions more clearly. Side-lobe levels are very good with nearly $-50 \mathrm{~dB}$ in the frequency domain and around $-25 \mathrm{~dB}$ for range.
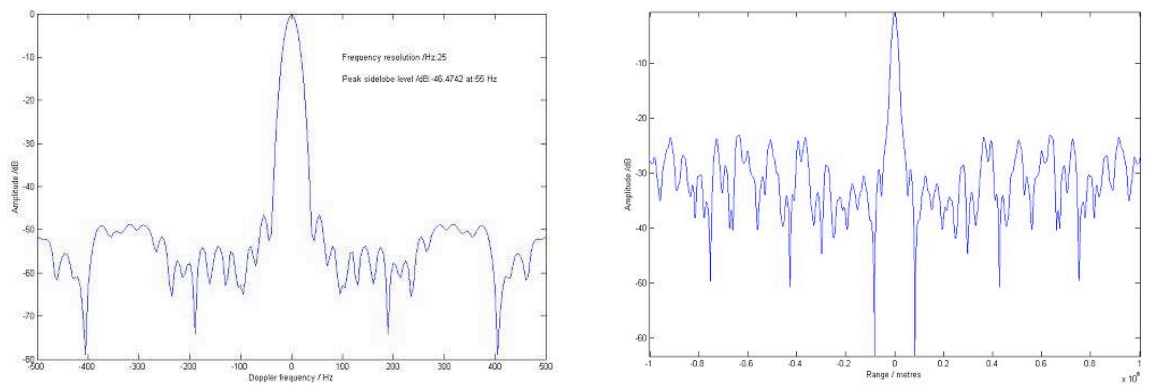

Figure 4. $\quad$ Range (left) and Doppler resolution (right) for the BBC radio 4 transmissions.

If a number of speech collections are analysed then we see that performance is not consistent. This is illustrated in Table 1 which shows the bandwidth in $\mathrm{kHz}$ for ten waveform samples.

The bandwidth is seen to vary from $500 \mathrm{~Hz}$ to $22.2 \mathrm{kHz}$. This is important in two respects. The first is that by no means all of the $150 \mathrm{kHz}$ modulation bandwidth is being used (in this case it is only $15 \%$ of the available bandwidth). Secondly, as the bandwidth is a function of time the performance of the radar system will also be a function of time.

So far only 'self-ambiguity' has been considered. This has been defined as the output of the matched filter response of the direct signal. Hence it may be thought of as a condition that mimics the performance of a monostatic radar system with the same waveform. In effect this yields the best possible range and Doppler resolutions with any given waveform. However, in PCL and more generally in bistatic radar the relative positions of target, transmitter and receiver govern the actual resolutions that can be achieved. Here we use the formulation presented in [21] to compute the bistatic ambiguity function for the example pre- 


\begin{tabular}{c|c} 
Collection Number & Bandwidth $/ \mathrm{kHz}$ \\
\hline 1 & 22.2 \\
2 & 0.5 \\
3 & 14.8 \\
4 & 9.1 \\
5 & 10.1 \\
6 & 0.8 \\
7 & 2.2 \\
8 & 4.2 \\
9 & 1.0 \\
10 & 5.3 \\
\hline Average Bandwidth $/ \mathrm{kHz}$ & 8.0
\end{tabular}

Table 1. Bandwidth variation of speech waveforms

sented in part 1 of this chapter that uses the transmitter at Alexander Palace with the receiver located at University College London. From the bistatic form of the ambiguity function this can be written as:

$$
\begin{array}{r}
\left|\psi\left(R_{R H}, R_{R a}, V_{H}, V_{s}, \theta_{R}, L\right)\right|= \\
=\mid \int_{-\infty}^{+\infty} s_{t}\left(t-\tau_{a}\left(R_{R a}, \theta_{R}, L\right)\right) \cdot s_{t}^{*}\left(t-\tau_{H}\left(R_{R H}, \theta_{R}, L\right)\right) \\
\left.\cdot e^{j\left(2 \pi f_{D H}\left(R_{R H}, V_{H}, \theta_{R}, L\right)-2 \pi f_{D a}\left(R_{R a}, V_{s}, \theta_{R}, L\right)\right) t} d t\right|^{2}
\end{array}
$$

where $R_{R H}$ and $R_{R a}$ are the hypothesised and actual ranges (delays) from the receiver to the target; $V_{H}$ and $V_{a}$ are the hypothesised and actual radial velocities of the target with respect to the receiver; $f_{D H}$ and $f_{D a}$ are the hypothesised and actual Doppler frequencies; $\theta_{R}$ is the angle from the receiver to the target with respect to 'North'; $L$ is the length of the baseline formed by the transmitter and receiver.

The expression assumes the reference point of the PCL geometry to be the receiver and is essentially a straight change of variables from the equation for the self ambiguity function. The important difference is that the geometrical layout of the transmitter, receiver and target are now taken into account. This can have a significant effect on the form of the ambiguity function and the resulting range and Doppler resolutions. Indeed if a target crosses the bistatic baseline all resolution in range and Doppler is lost.

Variations in range resolution can be distilled from the ambiguity 'traffic light' plot of Figure 5. Here the range resolution only is being plotted as a function of target position. The green colour span corresponds approximately to a range resolution of up to one and a half that 


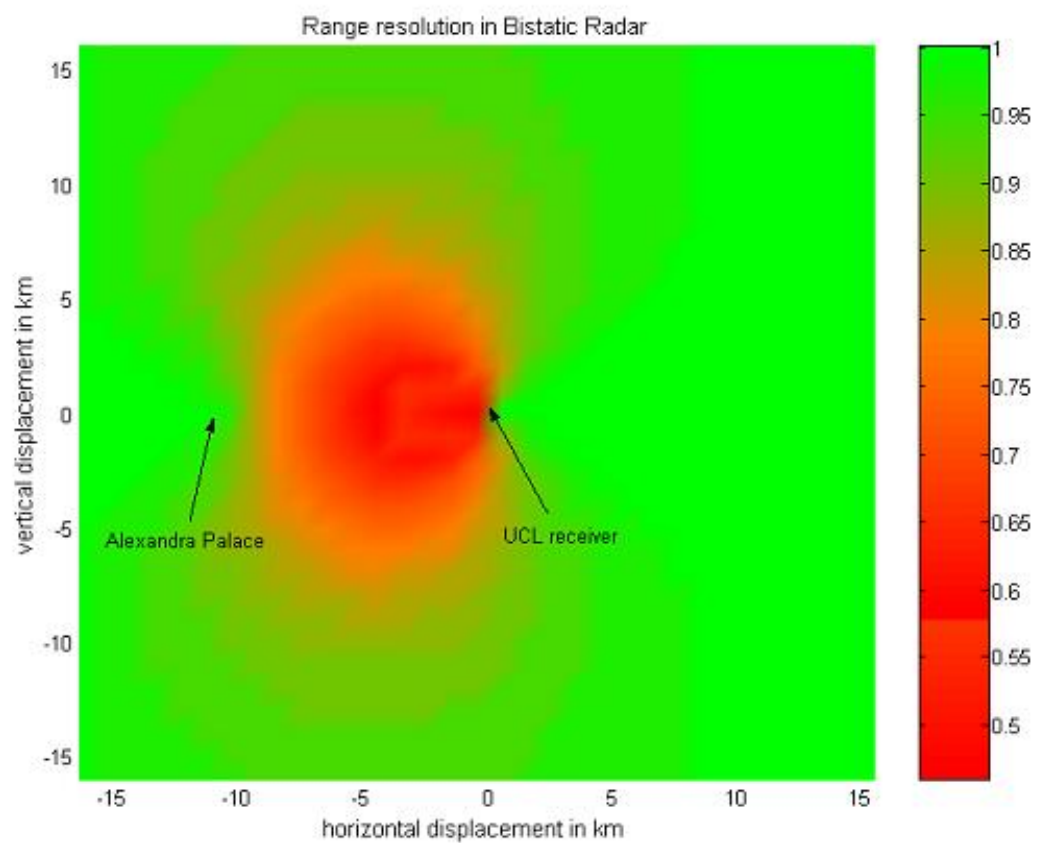

Figure 5. Traffic light plot of normalised range resolution variation for a bistatic PCL system.

of the self-ambiguity case. Amber corresponds to approximately a resolution degradation of $150 \%$ of that of the self-ambiguity case and red approximately greater than $200 \%$. The scale on the right hand side of the plot indicates this on a continuous colour change basis. This shows that there are significant areas where the resolution is severely degraded. There are several strategies for taking this into account in the design of the system. Two possibilities might be to either adjust the signal processing depending on the application or to simply declare a 'no-go' region where radar operation is not attempted. As the no-go area coincides with the direction of the directly received transmissions then this may be the preferred option as it aids the problem of suppression of the directly received signal.

Thus we have seen that PCL is a particular form of bistatic radar that has a number of attractive characteristics. It should not be thought of as a mature technology but more as an emerging one and extant systems are evidence of this. 


\section{Multistatic radar}

Most current radar systems are monostatic i.e. the transmitter and receiver are co-located. The performance of these forms of radar has been greatly enhanced by the advent of high resolution imaging, low sidelobe antennas, high speed digital signal processing and other technology improvements. However, it is well known that when a target is illuminated by electromagnetic radiation scattering occurs in all directions. A single receiver remotely located will only intercept a very small portion of this energy and much of the signal and its information is lost. Netted (or multistatic) topologies can overcome this limitation and offer the potential to extend the capabilities and performance of current radar systems.

Multisatic radar has some inherent advantages. For example spatial distribution of the nodes of the network enables the area to be tailored according to the specific application of interest. Additionally, it is possible to increase sensitivity, as more of the scattered energy (in the different directions) can be collected and hence detection performance improved. Target classification and recognition can also be enhanced, as the target is observed from different perspectives. Moreover, increased survivability and reliability is achieved because of the option of having 'silent' or passive operation of the receivers. These receivers can improve the location accuracy of possible jammers by fusing the information from the network nodes. Finally, if a single node of the network is lost it can still provide a level of (reduced) performance and the network is said to exhibit graceful degradation.

Here we extend the bistatic case to netted scenarios. The topology selected is the simple case of $\mathrm{N}$ transmitters and one common receiver, i.e. we have in effect a series of multiple bistatic geometries with varying baselines. The reason for this choice lies in the fact that it is convenient to reference all calculations to the single receiver, thus obtaining one unified form for the ambiguity function.

The analysis is based on the matched filtering performed at the receiver. Before proceeding to the mathematical background, it is necessary to state the assumptions made when modeling the system. These are:

i. The target is considered to be a slowly fluctuating scatterer.

ii. The transmitted signal after reflection by the target is multiplied by the factor $b$ which corresponds to the scattering characteristics of the target in the direction of the receiver. $b$ is assumed to be a Gaussian random variable when the number of scatterers is large and none is dominant. 
iii. The target's scattering properties do not change with the angle of view.

The transmitted modulation is common and has the following form:

$$
s(t)=\operatorname{Re}\left[u(t) \cdot \exp \left(j \omega_{c} t\right)\right]
$$

where $u(t)$ is the complex envelope of the signal and $\omega_{c}$ is the carrier frequency. The return signal will then be an addition of the $\mathrm{N}$ scattered signals from the target. An important assumption made at this point is that coherent processing of the raw data is feasible. This implies that the $\mathrm{N}$ echoes, which arrive at different time instances as the transmittertarget-receiver paths are different, can be processed jointly. This will involve storing a number of returns, aligning them and feeding them to the matched filter. The received signal is:

$$
r(t)=\Sigma_{i=1}^{N} \operatorname{Re}\left\{b_{i} \cdot u\left(t-\tau_{i}\right) \cdot e^{j \omega_{c}\left(t-\tau_{i}\right)}\right\}
$$

where $\tau_{i}$ is the time delay and $b_{i}$ is the multiplication factor. Taking into account assumption (iii), $b_{i}=b=$ constant.

It must be noted that the echoes that arrive at the receiver do not have the same intensity, as the propagation lengths of the waves are different. Thus, a weighting must be applied, according to the signal power. The weighting factors are calculated by the following set of equations:

$$
w_{i}=\frac{P_{R i}}{\max \left(P_{R i}\right)}, \quad i=1,2, \ldots N
$$

and

$$
P_{R i}=\frac{P_{T i} G_{T i} G_{r} \lambda^{2} \sigma_{B}}{(4 \pi)^{3}\left(R_{R} R_{T i}\right)^{2}}, \quad i=1 \ldots, N
$$

where the bistatic radar cross-section $\sigma_{B}$ is considered to be a constant. In the receiver, the weighted echoes are passed through a filter matched to the original transmitted signal. Following similar analysis to [21], and excluding $b$, the specific instance of the ambiguity function is given by:

$$
\text { Xnetted }=\left|\Sigma_{i=1}^{N} w_{i} X_{i}\right|^{2}
$$

where $X_{i}$ are the bistatic ambiguity functions for the different bistatic pairs that are formed.

Examining the scenario with $\mathrm{M}$ receivers and one common emitter there is a significant practical limitation when attempting to implement the previous methodology. It was assumed in that analysis that the scattered signals must be processed jointly. This is easier to achieve 
when there is a common receiver, whereas in this case all the receivers of the network must send their inputs to a central station for coherent processing and alignment of the signals. The real time requirement leads us to use an alternative approach. It will be assumed that each echo is processed in the corresponding receiver before being transmitted to a central unit. Thus, the processing has a distributed nature.

The outcome of this procedure will be the same as in the previous case in terms of the system's parameters (resolution and ambiguity), but not exploited via centralized processing as the ambiguity function seen by each receiver will be individually different.

The mathematical representation of the ambiguity function for this second method is now different. The matched filtering is performed for each echo and the final output will be a summation of the bistatic ambiguity functions of the various bistatic pairs. The following equation outlines this:

$$
\text { Xnetted }=\Sigma_{i=1}^{N} W_{i}\left|X_{i}\right|^{2} .
$$

Examining the general case where a number of nodes are spatially distributed, a combination of the two previous methods can be used. That is each of the $\mathrm{M}$ receivers in the network will accept all the scattered signals, originating from the $\mathrm{N}$ transmitting stations, creating a series of multistatic ambiguity functions. These will be then used as inputs in the last equation to construct the radar equation type ambiguity diagram. The outcome is:

$$
\text { Xnetted }=\Sigma_{j=1}^{M}\left(W_{j} \Sigma_{i=1}^{N} w_{i} X_{i}\right) .
$$

Assume the transmitted signal is a coherent train comprised of three rectangular pulses. The first example refers to the case where the target is close to the baseline $\left(\theta_{R}=-80^{\circ}\right)$ of one of the two bistatic pairs. For a simple bistatic radar it is well known that this scenario is detrimental to its resolution performance. The two baselines are set to $100 \mathrm{~km}$ and the target is stationary with $R_{R}=50 \mathrm{~km}$.

The left hand plot of Figure 6 represents the contour plot of the ambiguity function for the specific scenario. The horizontal axis is the range to the target and the vertical is the velocity. The right plot shows the cuts along these axes, in a position which corresponds to the actual values of the range and the velocity of the target. The width of the main peak corresponds to the resolution of the system and any additional peaks correspond to potential ambiguities.

The simulation result outlines that there is no significant improvement in the performance of the radar network, as compared to the bistatic 

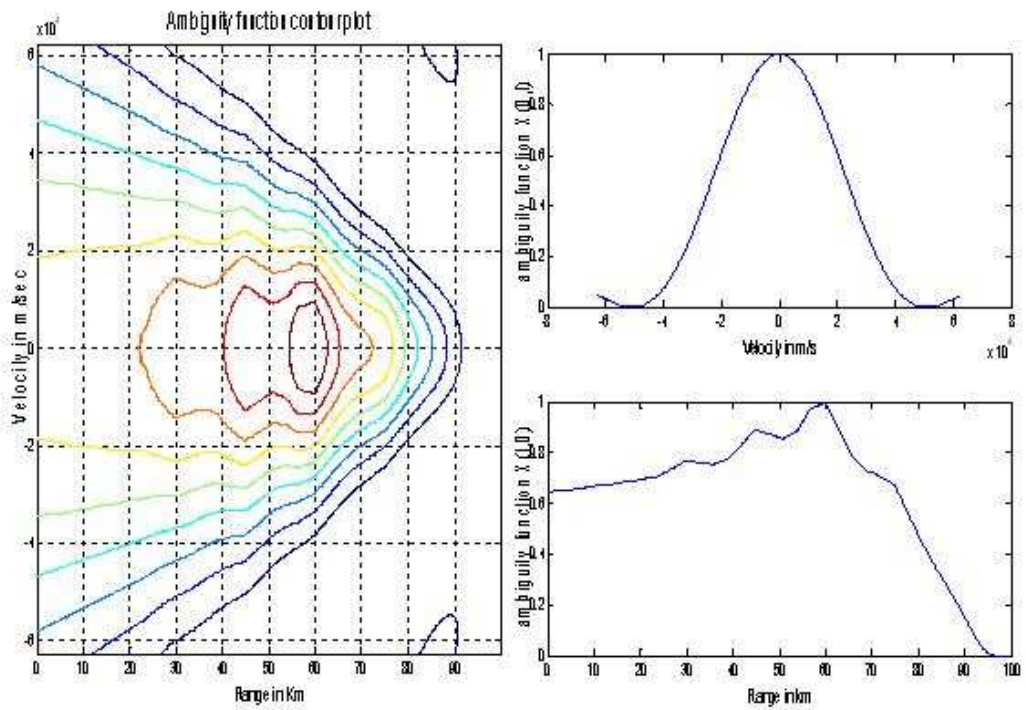

Figure 6. Contour plot and cuts along the velocity and range axis of the ambiguity function/bistatic pair dominance case.

radar. This is to be expected though, as for the specific position of the target one of the two bistatic pairs will be dominant. The echo which originates from the left transmitter will be much stronger than the one originating from the right one. Thus, the ambiguity function of the radar network will be dictated by the bistatic ambiguity function with the highest weighting factor.

There are scenarios though where the target is in such a position where none of the bistatic pairs is dominant. This is examined in the next example in a topology where the second transmitter is $100 \mathrm{~km}$ above the receiver. The angle $\theta_{R}$ of the target is $-60^{\circ}$.

The left plot represents the cuts of the bistatic ambiguity function of the first bistatic pair, in the absence of the second transmitter. Adding the emitter, and for this topology where the two weighting factors are comparable, the resolution in range and velocity has improved significantly. Moreover, the ambiguity peaks are suppressed.

Other degrees of freedom that can be varied are the baselines $L_{i}$. Returning to the geometry of the first example, where $\theta_{R}=-80^{\circ}$, the additional transmitter is placed in the position of the receiver, thus having a combination of monostatic and bistatic radar. In this way topologies can be tailored to meet application needs. However, it is most important to note that this must be balanced against the increased complexity of 

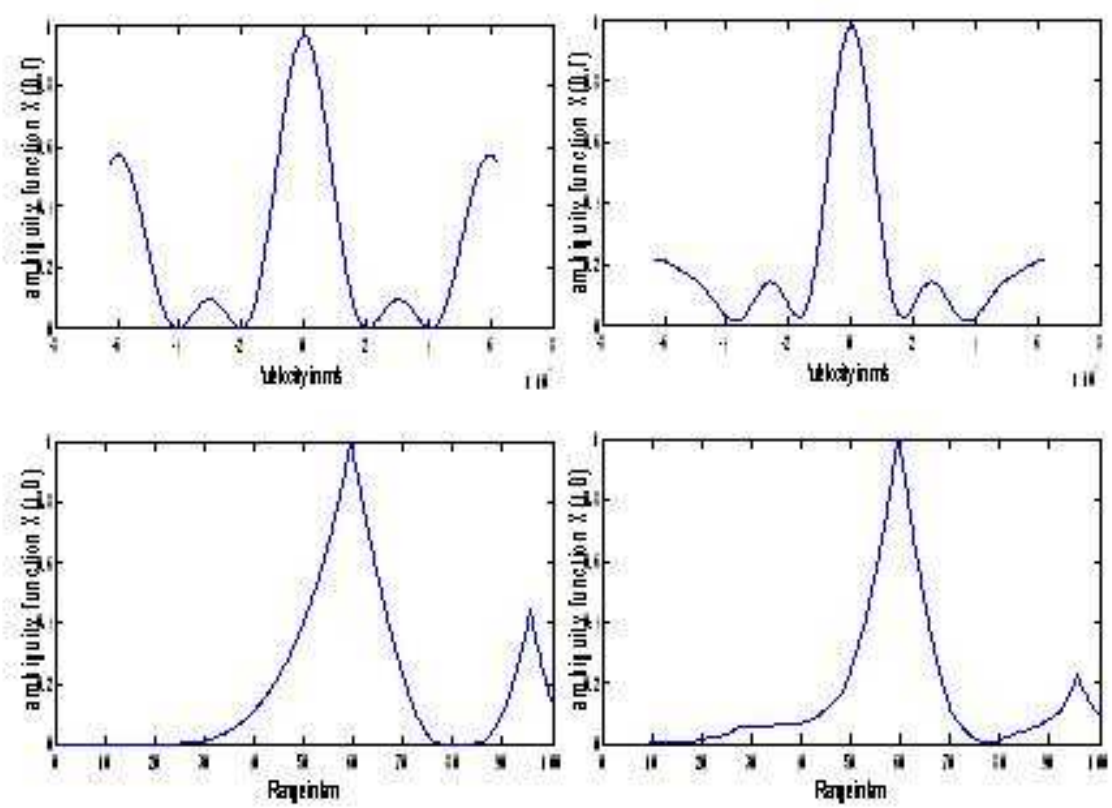

Figure \%. Bistatic and netted cuts of the ambiguity function balanced case

multistatic radar. The exception to this is PCL where more than one transmitter is exploited. Here the optimisation of system performance will be via intelligent signal processing. It is also unlikely that it will be possible to form a fully coherent distributed sensor in the way described above. However, the ability to capture and process signals from a variety of transmitters, situated in differing locations, transmitting on different frequencies provides a great deal of diversity that can be exploited to advantage.

\section{Conclusions}

Bistatic and multistatic radar have significant differences to their monostatic counterparts. This offers both advantages and disadvantages, particularly in applications addressing the issues of homeland security. The particular attraction of PCL is that it may be added to augment existing capability for target detection within home air space. A multiplicity of receiver sites as well as each receiver exploiting multiple transmitters results in a system that can be tailored to the prevailing conditions. It can take advantage of the space, time and frequency diversity inherent at each receiver site, and no expensive transmitter needs to be procured. Indeed in the VHF bands one can conceive of a sys- 
tem with equipment costs in the region of a few thousand dollars with a potential detection range of around $150 \mathrm{~km}$. For applications such as coastal surveillance for the protection of harbour areas and other points of vulnerability the lack of control over the emitted waveform may prove overly disadvantageous. Here there is a need to separate targets from (sea) clutter and generally resolutions are poor. However, as many of the targets of interest may well be moving at velocities considerably greater than that likely to be exhibited by clutter, Doppler processing might provide a route to success.

In summary, the essentials of bistatic and multistatic radar have been introduced and the resulting performance shows considerable promise for many application types. The additional attraction of the low cost of PCL makes this especially worthy of further attention.

\section{Acknowlegments}

The authors wish to thank Hervé Borrion for assistance in compiling this chapter into $\mathrm{LTT}_{\mathrm{E} X} \mathrm{X}$. We also recognise the contributions made by a number of students and in particular, Ioannis Papoutsis and Daniel O'Hagan.

\section{References}

[1] Willis, N.J. Bistatic Radar. Artech House, 1991.

[2] Baniak, J., Baker, G. Cunningham, A.M. and Martin, L. Silent Sentry passive surveillance Aviation and Space Technology, 7 June 1999

[3] Cherniakov, M., Nezlin, D. and Kubin, K. Air target detection via bistatic radar based on LEOS communication systems. IEE Proc Radar, Sonar and Navigation Vol.149, No.1, pp 33-38, February 2002.

[4] Giffiths, H.D., Baker, C.J., Baubert, J., Kitchen. N. and Treagust, M. Bistatic radar using spaceborne illuminator of opportunity. IEE Conf. Publ. Proc. RADAR 2002 Conference, Edinburgh No.490, pp 1-5, 15-17 October 2002.

[5] http://www.roke.co.uk/sensors/stealth/celldar.asp

[6] Sahr, J.D. and Lind, F.D. The Manastash Ridge radar: a passive bistatic radar for upper atmospheric radio science. Radio Science Vol.32, No.6, pp 2345-2358, November-December 1997

[7] Zoeller, C.L., Budge, M.C., Jr. and Moody, M. Passive coherent location radar demonstration Proceedings of the Thirty-Fourth Southeastern Symposium on System Theory pp 358-362, 18-19 March 2002.

[8] Griffiths, H.D. and Long, N.R.W. Television based bistatic radar. IEE Proceedings Vol. 133, Part F, No.7, pp 649-657, December 1986.

[9] Griffiths, H.D., Garnett, A.J., Baker, C.J. and Keaveny, S. Bistatic radar using satellite illuminators of opportunity Proc. RADAR'92 Conference, Brighton, IEE Conf. Publ. No.365, pp 276-279, 12-13 October 1992. 
[10] Griffiths, H.D. From a different perspective: principles, practice and potential of bistatic radar Proc. International Conference RADAR 2003, Adelaide, Australia, pp 1-7, 3-5 September 2003.

[11] Hawkins, J.M. An opportunistic bistatic radar Proc. RADAR'97 Conference, Edinburgh, IEE Conf. Publ. No.449, pp 318-322, 14-16 October 1997.

[12] Trizna, D. and Gordon, J. Results of a bistatic HF radar surface wave sea scatter experiment Proc. IGARSS '02' Vol.3, pp 1902-1904, 24-28 June 2002.

[13] Greneker, E.F. and Geisheimer, J.L The use of passive radar for mapping lightning channels in a thunderstorm. Proc. IEEE Radar Conference pp 28-33, 5-8 May 2003

[14] Yakubov, V.P., Antipov, V.B., Losev, DN. and Yuriev, I.A. Passive radar detection of radioactive pollution Application of the Conversion Research Results for International Cooperation SIBCONVERS '99. The Third International Symposium, Volume 2, pp 397-399, May 18-20, 1999.

[15] Howland, P. E. Target tracking using television based bistatic radar IEE Proc radar, Sonar and navigation, Vol 146, No 3, pp 166-174, 1999.

[16] Meyer, M. and Sahr, J.D. Passive coherent radar scatter interferometer implementation, observations and analysis Radio Science Vol.39, RS3008, doi: 0.1029/2003RS002985, May 2004.

[17] Herman, S. and Moulin, P.M. A particle filtering approach to passive radar tracking and automatic target recognition IEEE Aerospace Conference Proceedings, 2002. Vol.4, pp 1789-41808, 9-16 March 2002.

[18] Elirman, L.M. and Lanterman, A.D. A robust algorithm for automatic target recognition using passive radar Proceedings of the Thirty-Sixth Southeastern Symposium on System Theory, 2004 pp 102-106, March 14-16, 2004.

[19] http://www.bbc.co.uk/reception/

[20] http://www.sitefinder.radio.gov.uk/

[21] Tsao, T., Varshent, P., Weiner, D. and Schwarzlander, H. Ambiguity function for a bistatic radar IEEE Trans Aerospace and Electronic Systems, Vol. 33, No. 3, pp 1041-1051, 1997. 